\title{
Discrete Event Simulation of Patient Admissions to a Neurovascular Unit
}

\author{
Hahn-Goldberg, S., PhD ${ }^{1 *}$; Chow E., MS ${ }^{1}$; Appel E. MHEcon'; Ko F.T.F., BASc ${ }^{2}$; \\ Tan P., BASc ${ }^{2}$; Gavin M.B., BASc ${ }^{2}$; Ng T., BASc ${ }^{2}$; Abrams H.B., MD ${ }^{1,3,4}$; \\ Casaubon, L.K., MD MSc ${ }^{3,4}$ and Carter MW PhD ${ }^{2}$ \\ ${ }^{1}$ Centre for Innovation in Complex Care, University Health Network, Toronto, \\ Ontario, Canada, \\ ${ }^{2}$ Mechanical and Industrial Engineering, University of Toronto, Toronto, Ontario, \\ Canada, \\ ${ }^{3}$ University Health Network, Toronto, Ontario, Canada, \\ ${ }^{4}$ University of Toronto, Toronto, Ontario, Canada
}

Submitted October 2013. Accepted for publication June 2014.

\begin{abstract}
Evidence exists that clinical outcomes improve for stroke patients admitted to specialized Stroke Units. The Toronto Western Hospital created a Neurovascular Unit (NVU) using beds from general internal medicine, Neurology and Neurosurgery to care for patients with stroke and acute neurovascular conditions. Using patient-level data for NVU-eligible patients, a discrete event simulation was created to study changes in patient flow and length of stay pre- and post-NVU implementation. Varying patient volumes and resources were tested to determine the ideal number of beds under various conditions. In the first year of operation, the NVU admitted 507 patients, over $66 \%$ of NVU-eligible patient volumes. With the introduction of the NVU, length of stay decreased by around $8 \%$. Scenario testing showed that the current level of 20 beds is sufficient for accommodating the current demand and would continue to be sufficient with an increase in demand of up to $20 \%$.
\end{abstract}

Keywords: simulation modeling, stroke unit, healthcare, operations research

\section{INTRODUCTION}

Studies have shown that stroke unit care is associated with reduction of patient death and dependency [1, 2, 3, 4]. A systematic review by the Stroke Unit Trialists' Collaboration found that stroke unit care showed reductions in the odds of death and institutionalized care [5]. Chen et al. found that the average length of stay (LOS) of acute stroke patients was shortened after the implementation of a stroke unit [6]. Kapral et al. [7] assessed the effect of the Ontario Stroke System and found that processes of

*Corresponding author: Shoshana Hahn-Goldberg, The Centre for Innovation in Complex Care, 200 Elizabeth Street, Rm GNU403, Toronto, ON, M5G 2C4, e-mail: shoshana.hahn-goldberg@uhn.ca, phone: 416939 1507. Other authors: eric.khc@gmail.com, eva.appel@uhn.ca, frnklnk@gmail.com, peter.tan@utoronto.ca, bernadettegavin@gmail.com, terry.c.ng@gmail.com, howard.abrams@uhn.ca, leanne.casaubon@uhn.ca, carter@mie.utoronto.ca. 
care and outcomes after stroke were improved. Research has shown that stroke patients who receive appropriate interventions in a timely fashion have better outcomes [8], and Lahr et al. [9] found that an increased number of stroke patients received the recommended interventions when treated at a centralized stroke unit. The Clinical Effectiveness \& Evaluation Unit at the Royal College of Physicians of London created a set of standards for evaluating stroke units. One key indicator included in the standards is whether or not stroke patients were treated for at least $90 \%$ of their stay in the stroke unit [10].

In 2009, the Canadian Stroke Network published best practice recommendations for the implementation of a stroke unit, highlighting the ideal ratio of resources (e.g., beds, nurses, rehabilitation specialists) to patients, summarized from published ratios based on clinical implementations of stroke units [11]. Other countries and organizations have also published guidelines for implementing stroke units [12, 13, 14]; however, there is little guidance on how to implement a stroke unit while considering patient flow in related units that share similar resources.

In response to the evidence of improved outcomes for stroke patients in designated stroke units, many centres are looking at centralizing stroke care in stroke units, and there is research studying where and how to locate and organize stroke units $[15,16$, 17]. In Toronto, it was mandated to create three stroke regions to centralize stroke care within those specific hospitals' stroke units [18].

The Toronto Western Hospital (TWH), an acute care hospital and regional stroke centre in Toronto, Canada, created a Neurovascular Unit (NVU) in September 2011 - a next generation stroke unit for stroke and acute neurovascular conditions. TWH is a 272-bed academic health science centre which serves the population of downtown Toronto. As an estimate of volumes, the Emergency Department (ED) sees 60,000 patients annually. Patients were considered to be NVU-eligible based on their most responsible diagnosis (a list of ICD10 codes of the most responsible diagnoses for a patient's admission to hospital representing stroke and other acute neurovascular conditions was compiled to determine which patients would benefit and should be treated in the NVU). NVU patients could take complex paths through any number of units before entering into the care of the NVU. A majority of patients entered through the ED and were then treated on a selection of wards: General Internal Medicine (GIM), the Intensive Care Unit (ICU), the Neuro-Critical Care Unit (NCCU), Neurosurgery, and/or Neurology. Prior to the implementation of the NVU, stroke patients at TWH received care primarily from the GIM, Neurology, or Neurosurgery services. With limited resources, beds were needed from these services to implement the NVU. In order to create a 20-bed NVU, ten beds were allocated from GIM, five were allocated from Neurology, and five from Neurosurgery. Twenty beds were chosen using estimates of demand from previous years in an ad-hoc manner. Prior to this study, it was unclear, under resource constrained conditions, how internal bed allocation would influence the operational effectiveness of the NVU, as well the units whose beds were allocated to create the NVU. Furthermore, increased stroke patient volumes were expected, as stroke patients started being redirected from nearby hospitals without stroke units, in accordance with the mandate set within the three Toronto stroke regions during the 
study time frame (in late 2012). Prior to this study, the ability of the NVU to accommodate these external demands was unknown. This study aimed to investigate the effects of bed allocation on the operational effectiveness of the NVU and the hospital overall.

In this study, a discrete event simulation model was created to study the operational effects of an NVU implementation, based on the NVU experience at TWH and to confirm whether or not the decisions made by rule of thumb when designing the NVU were appropriate. Discrete event simulation has been used in health care for the past 40 years as a decision support tool that can help determine the most efficient use of resources $[19,20]$. In stroke care, simulation modeling has been used to support integrative planning for local stroke services [21] and to study the most appropriate staffing levels of specific stroke units [22]. We hypothesized that a simulation model depicting patient flow through the hospital units in question would allow for prediction of the best use of available resources. In particular, the creation of a simulation model and accompanying statistical analysis accomplishes two main goals: (1) It allows for easy visualization of the operational effects of the NVU on the other wards effected by its introduction and on the NVU-eligible patients, (2) It allows for potential generalizability of the model to other stroke units in the future. This model may be used as a future planning tool to support hospitals in effectively implementing a stroke unit.

\section{METHODS}

\subsection{Data Sources}

Actual bed history data for all NVU-eligible patients, determined by most responsible diagnosis (see Table 1), from 12/31/2009 to 09/26/2012, were used to create two discrete event simulations of patient flow pre- and post-NVU implementation. PreNVU was defined as patients discharged by September 6, 2011, while Post-NVU was defined as patients admitted by November 1, 2011. Patient data between Pre-NVU and Post-NVU, considered transition data, were discarded. During this transition time, service levels were deemed to be inconsistent, as it was the period of training and adjustment for the NVU. For the purposes of this study, NVU-eligible patients were divided into two patient types - stroke and neurovascular (NV). Classifications were made by grouping the ICD-10 codes as defined by the hospital.

Actual bed history data for all non NVU-eligible patients who visited the GIM and Neurology/Neurosurgery units, the units which allocated beds in order to create the NVU, was also collected. The inpatient visits were grouped into the same pre-NVU and post-NVU time periods as described above. All data were extracted from the electronic patient record at TWH. For each patient, we collected the LOS in each nursing unit that they were seen in as well as the LOS in the emergency department (if applicable). In addition, we also collected the most responsible diagnosis associated with their stay.

\subsection{Patient Flow}

In the discrete event simulation of the patient flow of NVU-eligible patients at TWH, each ward was grouped into one of five main units: (1) ED, (2) GIM, (3) NCCU, (4) Neurology/Neurosurgery, and (5) others. Patients exited the hospital via three paths: 
Table 1. Patient classification by most responsible diagnosis ICD10 codes

\begin{tabular}{|c|c|c|}
\hline Description & Code & $\begin{array}{c}\text { Patient } \\
\text { Type }\end{array}$ \\
\hline \multicolumn{3}{|l|}{ Benign neoplasm of } \\
\hline carotid body & D35.5 & NV \\
\hline Vascular myelopathies & G95.1 & NV \\
\hline \multicolumn{3}{|l|}{ Retinal artery and vascular } \\
\hline occlusion (excluding H34.1 below) & H34. $(0,2,8,9)$ & NV \\
\hline Other SAH & $\mathrm{I} 60.8^{*}$ & NV \\
\hline \multicolumn{3}{|l|}{ Cerebral infarction due to } \\
\hline cerebral venous thrombosis & I63.6* & NV \\
\hline Arterial occlusion/stenosis & I65 & NV \\
\hline Other cerebrovascular diseases & I67 & NV \\
\hline Sequelae of cerebrovascular disease & I69 & NV \\
\hline \multicolumn{3}{|l|}{ Arteriovenous and other malformations } \\
\hline of precerebral and cerebral vessels & Q28.(0,1,2,3) & NV \\
\hline Transient Ischemic Attack [TIA] & G45 & Stroke \\
\hline Subarachnoid Hemorrhage $[\mathrm{SAH}]$ & $\mathrm{I} 60$ & Stroke \\
\hline Intracerebral Hemorrhage [ICH] & I61 & Stroke \\
\hline Ischemic Stroke & I63 & Stroke \\
\hline Acute Stroke - unspecified & I64 & Stroke \\
\hline Central Retinal Artery Occlusion & H34.1 & Stroke \\
\hline
\end{tabular}

home, death, and transfer. To determine flow paths and proportions, data were processed to match each ward into a major unit of ED, GIM, Neurology, Neurosurgery, and NCCU, and the percentages of NVU-eligible patients flowing from each unit to every other unit were calculated. Figure 1 provides a screenshot of the post-NVU simulation model depicting the patient flow of NVU-eligible patients. The most dominant pathways are from the ED to a GIM unit, a neurology unit, or the NVU.

\subsection{Statistical Modeling}

The simulations of NVU-eligible patients were created using Simul8 discrete event simulation software [23]. All data processing was performed using Microsoft Excel, and all statistical distributions were fitted with@RISK [24] statistical software. @Risk ensures that the distributions used to represent the real data are within $95 \%$ accuracy. Simul8 allows the creation of a visual representation of patient flow through the various units in the hospital using fitted distributions and mathematical representations of relationships between various parameters. Simulations use much probability and are as such not $100 \%$ accurate and must be sufficiently validated to ensure that the results are 


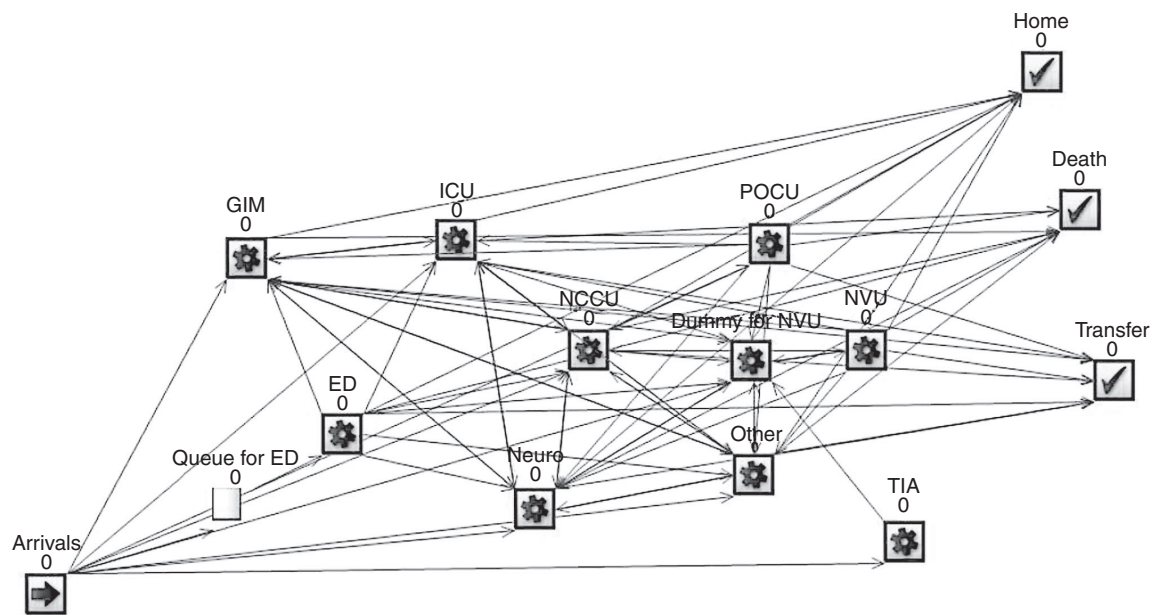

Figure 1. Post-NVU simulation screenshot. ED is Emergency Department, ICU is Intensive Care Unit, GIM is a General Internal Medicine unit, Neuro is a Neurology or Neurosurgery unit, NCCU is a Neuro-Critical Care Unit, POCU is Post-Operative Care Unit, NVU is Neurovascular Unit, TIA is the Trans-Ischemic Attack Unit.

usable. Simul8 automatically calculates many metrics including average and maximum utilization of each unit, time in the system per patient, and others which will be referenced in the following sections. Model input parameters included (1) inter-arrival rate distribution of new patients into the system, (2) flow paths and proportions of patients into and out of each ward, (3) LOS distributions for each of the five main units, and (4) bed capacities for each of the five main units. Each of the five main units may comprise several hospital wards and the number of beds available in each unit was calculated by totaling the beds for any wards in that unit. The post-NVU simulation model contained routing instructions for the simulated patients sent to the NVU at times of full capacity; these patients would be redirected to the Neurology ward in the discrete event simulation, reflecting actual practice at TWH.

Inter-arrival rates were determined from the duration of time between consecutive NVU-eligible patients arriving at the hospital. Each inter-arrival time was calculated from the historical data and statistical distributions were fitted to the inter-arrival values using@Risk, and fit was evaluated using the $\mathrm{R}^{2}$ statistic. To determine LOS distributions, all data entries were filtered by unit, and the LOS values of all visits to that unit were collected. Statistical distributions were then fitted to the LOS values using@Risk. The simulation models used the visual logic function in Simul8 to read in LOS distribution parameters and flow proportions from external files.

\subsection{Model Validation}

The simulation models presented above were validated following the process presented by Macal [25]. Input and output data were validated by checking the agreement of the 
data with the original data sources and ensuring the appointments that occurred at the hospital also occurred in the models and the durations of the various stages matched the durations in the original data. Requirements validation was conducted by several parties independently to ensure all the constraints worked as expected. Face validation was conducted by subject matter experts at the TWH to ensure the output of the models was realistic given the known assumptions present in the models in question.

In addition to the above validation processes, process validation was also conducted on the simulation model. The validity of the simulation models was assessed through two additional simulations that were modeled deterministically rather than stochastically. Patients arrived at the exact admission times in the data, followed the exact path taken during their stay according to the visit area data, and stayed in each unit exactly as the LOS data indicated. The deterministic models were set up to run a length of time such that all patients in the data were able to enter the system. The stochastic models were set up to run the same amount of time, and output data were collected for both sets of models. The deterministic results were then compared to the $95 \%$ confidence interval of the stochastic results. The metrics considered were the number of arrivals to the system, the number of patients serviced by the various units, and the average utilization of the units.

\subsection{Experimental Design}

Using the simulations, four sets of experiments were conducted to study the following key questions:(1) how much demand can the system handle at its current capacity, (2) how much bed capacity should the NVU have, (3) what are the impacts if the LOS in the NVU is reduced, and (4) what are the overall impacts of the NVU implementation on the bed capacity of the various units. In each experiment, a single factor or variable was modified and simulation outputs were collected and analyzed.

For the first three sets of experiments, the following metrics were observed: average NVU utilization (number of NVU beds occupied); average NVU percent utilization ([average NVU utilization]/[maximum NVU capacity]); maximum NVU utilization (maximum number of NVU beds occupied at any point in time); maximum NVU percent utilization ([maximum NVU utilization]/[maximum NVU capacity]); redirections at full capacity (number of patients redirected to neurology when the NVU was full); and percent redirected ([redirections at full capacity]/[number of patients requiring services from the NVU]).

For the last set of experiments, answering the question about overall impacts of the implementation of the NVU, discharge type, total time in system, average utilization for each unit, and maximum utilization for each ward were observed.

Since simulations have inherent probability, we perform multiple runs and report on the mean and confidence interval of all results. In our case, the following numbers of runs were deemed sufficient: The trial for the Pre-NVU model consisted of 45 runs of 14616 hours each. The trial for the Post-NVU model consisted of 68 runs of 7870 hours each. The amount of data available for the pre-and post-NVU models was different and hence required differing number of runs. 
To determine the level of demand the system can potentially handle, simulated interarrival rates were increased by 5\%,10\%, $15 \%$ and $20 \%$. To determine the optimal bed capacity of the NVU for the current as-is system, the simulated number of available beds was varied between 15 and 30. To determine the impacts on the system if a reduction in LOS for NVU patients hospitalization was achieved, simulated LOS was reduced by $12,24,36$, and 48 hours.

\subsection{Non NVU-Eligible Patients}

Descriptive statistics were used to determine the operational effects of the NVU on the GIM and Neurology/Neurosurgery units that allocated bed resources to create the NVU. The average and standard deviation of the LOS was calculated for non-NVU patients visiting the GIM and Neurology/Neurosurgery units both pre- and post-NVU implementation.

\section{RESULTS}

The actual data showed 1312 NVU eligible patients in the pre-NVU collection period and $640 \mathrm{NVU}$ eligible patients in the post-NVU collection period. Of these patients, 376 (28.65\%) had a most responsible diagnosis classified as NV pre-NVU and 184 (28.79\%) had a most responsible diagnosis classified as NV post-NVU. The remainder of NVU eligible patients had a most responsible diagnosis classified as Stroke.

\subsection{Patient Flow}

The entry points pre- and post-NVU were very similar, with a majority of simulated patients (63\% and 65\%, respectively) entering through the ED, and $17 \%$ and $19 \%$, for pre-and post-NVU respectively, coming in for surgery. The remaining patients entered directly to a ward. After the ED, there was a large difference in simulated patient flow pre- and post-NVU, with fewer patients going to GIM and Neurology post-implementation because they went to the NVU instead. Admissions to GIM dropped from $47 \%$ pre-NVU to $7 \%$ post-NVU, and admissions to Neurology dropped from $17 \%$ to $9 \%$.

\subsection{Statistical Modeling}

The inter-arrival rate of patients into the system was represented by an exponential distribution with parameter of 11.354 hours pre-NVU and a parameter of 12.306 hours post-NVU. There were no time dependencies in the means of these distributions as there was no statistical difference in inter-arrival rate based on day of the week or time of the day.

Pre-NVU, the GIM unit contained 98 beds and neurology/neurosurgery contained 67. In order to create the NVU, 10 beds were re-allocated to the NVU from GIM, 5 from Neurology, and 5 from Neurosurgery. As a result, the bed distribution post-NVU was 20 beds in the NVU, 88 in GIM and 57 in Neurology/Neurosurgery. Log Normal or Pearson5 distributions were found to match well to the actual LOS data on the various units. 


\subsection{Model Validation}

All relevant output metrics of the actual collected TWH data fell within the 95\% confidence interval of the simulation. In particular, the following values were measured for both the pre- and post-NVU models: (1) number of patients entered, (2) jobs completed by unit, (3) average utilization by unit, (4) maximum utilization by unit, (5) maximum use at one time by unit, (6) number of redirections from the NVU (post-NVU model only), and (7) percent of redirections from the NVU (post-NVU model only). A sample of the mean values of the base post-NVU model is provided in Table 2 . The simulation models were considered to be appropriate representations of the patient flow and operations of the NVU and connecting units, and could be used to run further experiments. The experts reviewing the face validity of the model were satisfied with the model performance.

\subsection{Experimental Results}

The average NVU utilization in the discrete event simulation was 10.88 (54.4\%), given the base capacity of 20 beds, base patient arrival rates, and base processing times. The maximum utilization at any given point in time was 19.83 (99.1\%). The NVU in the discrete event simulation treated 441.25 simulated patients. An additional 3.82 simulated patients requested service, but were sent to neurology because the NVU was full. Overall, 640 simulated NVU-eligible patients were seen in the simulation by the hospital overall.

For the first set of experiments, the demand of simulated NVU-eligible patients was increased. The average utilization increased from 54.4\% at the baseline to $64.7 \%$ with a $20 \%$ increase in arrivals. The maximum utilization increased from $99.1 \%$ to $100 \%$. Additionally, the number of redirected patients increased from $3.82(0.9 \%)$ at the base arrival rate, to about $20.09(3.8 \%)$ at a 20 percent increase in arrival rate.

For the second set of experiments, the number of simulated bed resources in the NVU was varied from 15 beds to 30 beds. Average utilization of the NVU increased slightly as more beds were added. At about 20-bed capacity, the rate of maximum utility increase dropped off significantly (see Figure 2). Moreover, maximum utilization at any point increased perfectly at a one to one rate until 20-bed capacity was reached, after

Table 2. Base results of the post-NVU model

\begin{tabular}{lcc}
\hline Ward & Measure & Base Result \\
\hline NVU & Average use & 10.88 patients \\
NVU & Average \% utilization & $54.4 \%$ beds \\
NVU & Maximum use & 19.83 patients \\
NVU & Max \% utilization & $99.1 \%$ beds \\
NVU & Redirections at full capacity & 3.82 patients \\
NVU & Total requests & 441.25 patients \\
NVU & $\%$ redirected & $0.9 \%$ patients \\
System & Arrivals & 639.56 patients \\
\hline
\end{tabular}




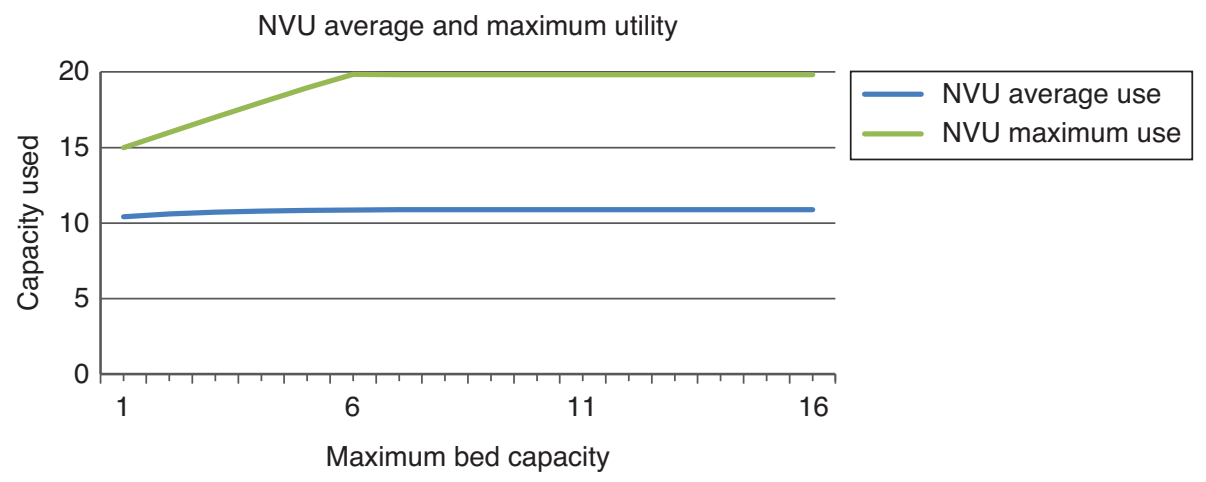

Figure 2. Utilization versus bed capacity.

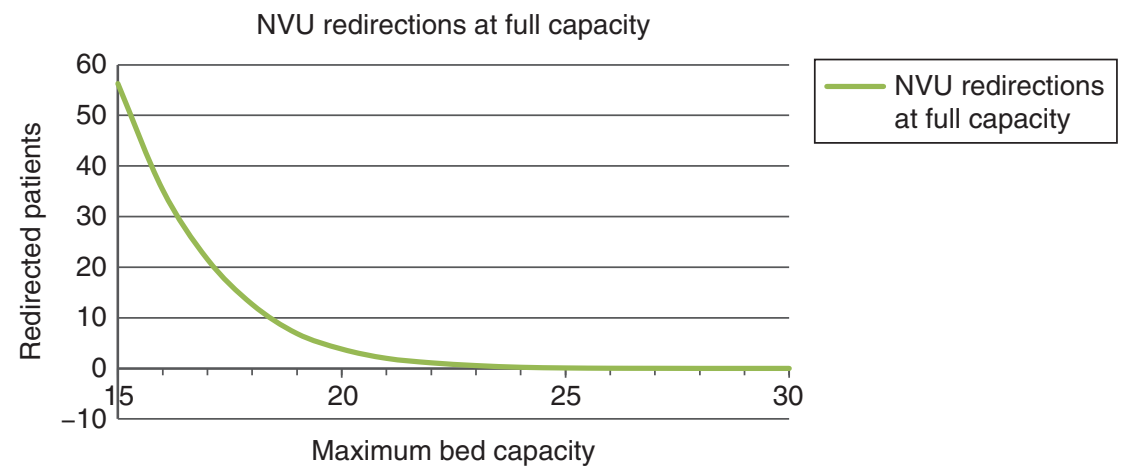

Figure 3. Redirections at full capacity versus bed capacity.

which it remained constant. Patients requiring NVU beds were sent to the neurology ward if the NVU was full. The percentage of patients redirected decreased rapidly as more beds were added from the minimum capacity of 15 . However, the rate of improvement in the number of redirections decreased with diminishing returns. By the 20-bed capacity, both the percent of redirected patients and the rate of improvement were very small, as shown in Figure 3 . The point where diminishing returns began to exceed improvements was 20 beds, which was the bed capacity in the actual NVU. Although some of these results are intuitive and expected, it was important to the physicians planning the NVU to have them validated in such simulation.

The third set of experiments involved decreasing the simulated total LOS for patients in the NVU. There was a linear decrease in average bed utilization rate as the average LOS for patients in the NVU decreased. Average utilization decreased from $54.4 \%$ at baseline to $43.0 \%$ with a decrease in LOS of 48 hours. Similarly, the maximum bed utilization rate also decreased linearly. Maximum utilization decreased from $99.1 \%$ at baseline to $90.2 \%$ with a reduction in LOS of 48 hours. The number of patients redirected due to the NVU being full decreased from 4.82 patients at baseline to 0.39 with a reduction in LOS of 48 hours. 
For the final set of experiments, the results of the pre- and post-NVU simulations were compared. After introducing the NVU, there were significant decreases in simulated utilization in the GIM and Neurology/Neurosurgery units, as patients were being admitted to the NVU instead. Average GIM utilization dropped from 7.4 to 0.7 patients and average neurology/neurosurgery utilization dropped from 8.6 to 1.6 patients post-NVU. The maximum utilization dropped from 15.9 to 4.1 and from 18.4 to 6.5 patients in GIM and neurology/neurosurgery, respectively. The efficiency of the system increased, as the average total LOS of NVU-eligible patients decreased from 300 hours to 276 hours post-NVU implementation.

\subsection{Non NVU-Eligible Patients}

There were a total of 13351 (417 patients per month) and 4802 (150 patients per month) actual visits of non-NVU-eligible patients to GIM and Neurology/Neurosurgery, respectively, in the 32-month pre-NVU period, and 3727 (373 patients per month) and 1394 (140 visits per month) visits in the 10-month post-NVU period. Although not statistically significant, the LOS in the hospital for patients admitted to GIM as well as Neurology/Neurosurgery decreased over time with the introduction of the NVU. Patients admitted to GIM had an actual average LOS of 219.8 hours pre-NVU, which reduced by 24 percent to 167.8 hours post-NVU. Similarly, the average LOS for patients admitted to Neurology/Neurosurgery decreased from 246.3 hours pre-NVU to 199.7 hours post-NVU. Furthermore, in patients admitted to both GIM and Neurology/Neurosurgery, there is a reduction in the standard deviation of LOS and the median LOS as well.

\section{DISCUSSION}

This study has demonstrated the value of using discrete event simulation approaches in planning for stroke unit implementation. The discrete event simulation model was able to confirm that the 20 beds re-allocated from GIM, Neurology, and Neurosurgery to the NVU was able to accommodate the demand under current conditions. Furthermore, the simulation confirmed that NVU-eligible patients previously admitted to GIM and Neurology/Neurosurgery were admitted to the NVU after it was implemented, thereby following best practice recommendations for stroke patients to be cared for on a stroke unit. Moreover, there were no adverse effects to non-NVU-eligible patients visiting units that allocated beds to create the NVU. In fact, the LOS for these patients decreased after the implementation of the NVU. Additionally, the outputs of the simulation models were deemed satisfactory by the physicians working in the NVU.

The NVU in the discrete event simulation treated 442 out of 640 simulated NVUeligible patients, showing that about $70 \%$ of eligible patients were treated in the NVU. Ideally, the physicians of the NVU would expect to see $80 \%$ of eligible patients treated in the NVU. The reasons that we would not expect to see $100 \%$ of eligible patients in the NVU are the following: (1) About 5\% of patients are assumed to be classified incorrectly as NVU-eligible via the administrative database. (2) One of the limitations to using hospital administrative data in the development of the simulation model is that the administrative data do not capture all the exclusion criteria for the NVU. For example, palliative patients or those with severe dementia who suffer a condition that 
would normally make them NVU-eligible are excluded and re-directed to GIM. This number is reflected accurately in the simulation and was confirmed by GIM and Neurology physicians. (3) Another 5\% of patients are either sent home or do not become stable enough to be transferred from the ICU. This number is also accurately represented in the simulation. However, there still remain another $10 \%$ of patients (the difference between the actual $70 \%$ and the expected $80 \%$ ) that should be treated in the NVU. Upon closer inspection of the actual data and discussions with neurologists, it was found that there remained a number of patients being treated in Neurology and Neurosurgery because beds in the NVU were being used by other services incorrectly. Efforts are being undertaken by the hospital to ensure that all NVU-eligible patients be treated in the NVU.

The discrete event simulation showed that there was room within the current capacity of 20 beds to accommodate a $20 \%$ increase in demand. However, with the improvement in system function, the $10 \%$ of NVU-eligible patients not currently treated in the NVU will soon be directed there. Thus the increased demand that can be accommodated from other hospitals with current NVU resources is closer to $10 \%$ or just under 100 patients per year.

Our preliminary analysis shows that an NVU is possible within an acute care hospital and can be implemented using existing resources. The NVU at TWH resulted in an observed decrease in LOS for the care of NVU-eligible patients. This observed decrease is likely a result of the introduction of the NVU; however, it is also possible that it is due to other hospital-wide interventions such as lean processes being adopted hospital-wide and/or other quality improvement initiatives. Before approaching the implementation of an NVU, hospitals should conduct a thorough analysis of their own NVU-eligible patients to determine their demand as well as what resources they currently use in their hospital. This will help them calculate how many patients they can expect to see in their NVU and how much volume will be removed by the introduction of the NVU and from where. This will help immensely in their planning.

The current simulation model only includes bed resources and NVU-eligible patients, but does not differentiate between stroke types and stroke severity or between acute and alternative level of care days in the hospital. Additionally, the effect of transitions at disposition, e.g., stroke rehabilitation, is also not included. More research is warranted to include additional NVU resources, such as staffing, and to evaluate aspects such as the impact of the transition to stroke rehabilitation and differences in stroke severity. Additionally, it will be important to determine whether the initial operational benefits of the implementation of the NVU have been sustained beyond one year. A separate study will examine whether the expected improvements in clinical outcomes have been achieved.

\section{CONCLUSION}

A Neurovascular Unit can be implemented within an acute care hospital using existing resources with minimal disruptions and no adverse effects to the operations of the hospital, as was done in the case of the NVU at the TWH. After implementation of the NVU, NVU-eligible patients previously treated in the GIM or Neurology and 
Neurosurgery wards, were treated in the NVU instead and received specialized neurovascular care. Additionally, the NVU at the TWH achieved decreased LOS for the care of NVU-eligible patients.

Literature shows that NVUs are a good choice for patient outcomes. The NVU at TWH was designed by rule of thumb and the analysis presented in this paper confirmed, retrospectively, that this NVU has been a good choice operationally as well. The analysis of the NVU at TWH showed that the average NVU utilization is currently under $60 \%$ in the discrete event simulation. As a whole, the analysis suggests that there is room to accommodate more than a $20 \%$ growth in the number of patients at the current capacity and processing time. The number of redirected patients expected to come with growth in demand is not of sufficient magnitude to justify an introduction of more beds to the NVU. In addition, the analysis suggests that if patients can be discharged home or transferred to a rehabilitation facility earlier, more beds will become available in the NVU, which can therefore serve a greater demand. However, further research is warranted to model the complexities of alternative level of care days, staffing resources, and their impact on clinical outcomes.

\section{ACKNOWLEDGEMENTS}

Funding for this research was provided in part by the Krembil Foundation.

\section{CONFLICT OF INTEREST}

The funding agreement for this study ensured the authors' independence in designing the study, interpreting the data, writing, and publishing the report. The authors have nothing to disclose and no conflicts of interest.

\section{REFERENCES}

[1] Fjaertoft H, Rohweder G, Indredavik B. Stroke unit care combined with early supported discharge improves 5-year outcome. Stroke, 2011, 42: 1707 - 11.

[2] Evans A, Perez I, Harraf F, Melbourn A, Steadman J, Donaldson N, Kalra L. Can differences in management processes explain different outcomes. Lancet, 2001, 358 (9293): 1586 - 92.

[3] Rotter T, Kinsman L, James E, Machotta A, Willis J, Snow P, Kugler J. The effects of clinical pathways on professional practice, patient outcomes, length of stay, and hospital costs. Eval Health Prof, 2012, 35 (1): 3-27.

[4] Cereda CW, Stadler C, Andreotti J, Pelloni L, Frangi J, Pifferini V, Bassetti C. Impact of implementation of a new semi-intensive stroke unit versus a mobile stroke team system - A prospective study within the neurocentro of Southern Switzerland. Clinical Neurophysiology, 2012, 123(10): e101.

[5] Stroke Unit Trialists' Collaboration. Collaborative systematic review of the randomized trials of organized inpatient (stroke unit) care after stroke. BMJ, 1997, 314: 1151-9.

[6] Chen LK, McClaran J, Buchan AM. Impact of acute stroke on hospital length of stay. Archives of Gerontology and Geriatrics, 2009, 49(1): e12-5.

[7] Kapral MK, Silver FL, Hall R, Stamplecoski M, O'Callaghan C, Tu JV. Effect of a provincial system of stroke care delivery on stroke care outcomes. CMAJ, 2013, 185(10): e483-91.

[8] Addo J, Crichton S, Bhalla A, Rudd AG, Wolfe CDA. Impact of implementation of evidence-based acute stroke interventions on survival: the South London Stroke Register. PLoS, 2013, 8(4): e61581. 
[9] Lahr M, Luijckx GJ, Vroomen P, van der Zee DJ, Buskens E. Proportion of patients treated with thrombolysis in a centralized versus decentralized acute stroke care setting. Stroke, 2012, 43: 1336-40.

[10] Hoffman A, Campbell J, Grant R, Lowe D, Rudd A, Stroke obot I, et all. National sentinel stroke audit phase II (clinical audit) 2008. Report for England, Walwsand Northern Ireland. London: Royal College of Physicians of London. 2009.

[11] Lindsay MP, Gubitz G, Bayley M, Hill MD, Davies-Schinkel C, Singh S, Phillips S, Canadian Stroke Strategy Best Practices and Standards Writing Group. Canadian best practice recommendations for stroke care. Ottawa (ON): Canadian Stroke Network; 2010, 85-98.

[12] National Collaborating Centre for Chronic Conditions (UK). Stroke: National Clinical Guideline for Diagnosis and Initial Management of Acute Stroke and Transient Ischaemic Attack (TIA). London: Royal College of Physicians (UK); 2008. (NICE Clinical Guidelines, No. 68.) Available from: http://www.ncbi.nlm.nih.gov/books/NBK53295/

[13] Intercollegiate Stroke Working Party. National clinical guideline for stroke, 4th edition. London: Royal College of Physicians, 2012.

[14] Jauch EC, Saver JL, Adams HP, Bruno A, Conners JJ, Demaerschalk BM, Khatri P, McMullan PW, Qureshi AI, Rosenfield K, Scott PA, Summers DR, Wang DZ, Wintermark M, Yonas H. Guidelines for the early management of patients with acute ischemic stroke. Stroke, 2013, 44: 870-947.

[15] Leira EC, Fairchild G, Segre AM, Rushton G, Froehler MT, Polgreen PM. Primary stroke centers should be located using maximal coverage models. Stroke, 2012, 43: 2417-22.

[16] Cramer SC, Stradling D, Brown DM, Carrillo-Nunez IM, Ciabarra A, Cummings M, Dauben R, Lombardi DL, Patel N, Traynor EN, Waldmen S, Miller K, Stratton SJ. Organization of a United States county system for comprehensive acute stroke care. Stroke, 2012, 43: 1089-93.

[17] Ringelstein EB, Chamorro A, Kaste M, Langhorne P, Leys D, Lyrer P, Thijs V, Thomassen L, Toni D. European stroke organization recommendations to establish a stroke unit and stroke center. Stroke, 2013, 44: 828-40.

[18] Implementing Stroke and Orthopaedic Best Practices in the Toronto Central LHIN: Analysis of System Wide Impacts. Hay Group, 2012.

[19] Jacobson S, Hall S, Swisher J. Discrete event simulation of healthcare systems. International Series in Operations Research and Management Science, 2006, 91:211-52.

[20] Gunal M, Pidd M. Discrete event simulation for performance modelling in health care: a review of the literature. Journal of Simulation, 2010, 4: 42-51.

[21] Bayer S, Petsoulas C, Cox B, Honeyman A, Barlow J. Facilitating stroke care planning through simulation modelling. Health Informatics Journal, 2010, 16(2): 129-43.

[22] Heinrichs M, Beekman R, Limburg M. Simulation to estimate the capacity of a stroke unit. Stud Health Technol Inform, 2000, 77: 47-50.

[23] Simul8. Simul8 Simulation Software 2012. Retrieved from: http://www.simul8.com/products/index.htm. Accessed: October 24, 2012.

[24] Palisade. @RISK:Risk Analysis Software using Monte Carlo Simulation for Excel 2012. Retrieved from: http://www.palisade.com/risk/?gclid=CPORyrq117MCFYw-Mgod-hEA3A. Accessed: October 23, 2012.

[25] Macal C. Model verification and validation. The Argone National Laboratory, University of Chicago, 2005. 



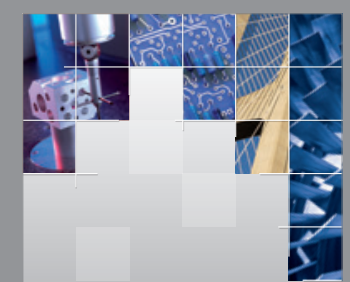

\section{Enfincering}
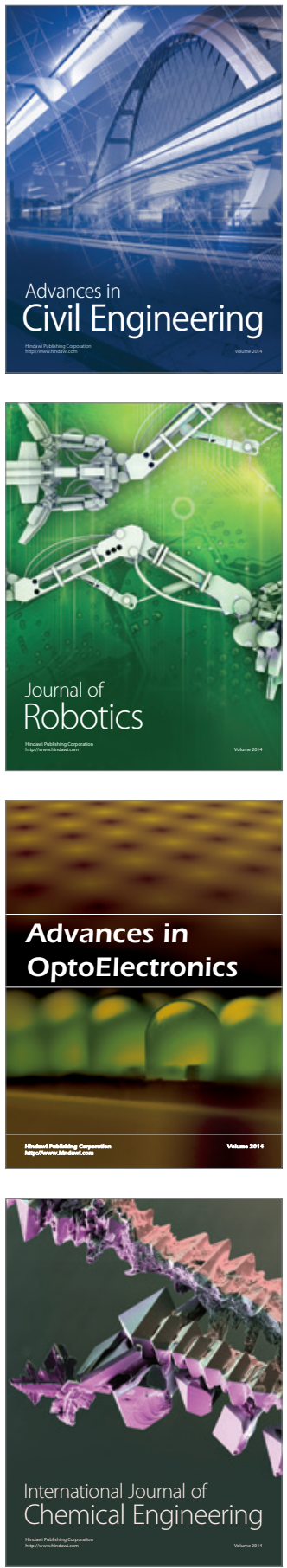

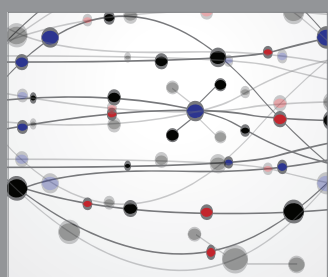

The Scientific World Journal

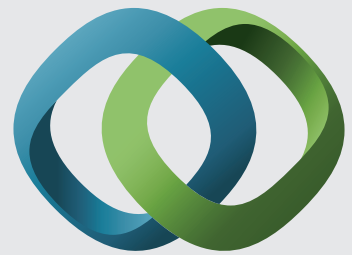

\section{Hindawi}

Submit your manuscripts at

http://www.hindawi.com
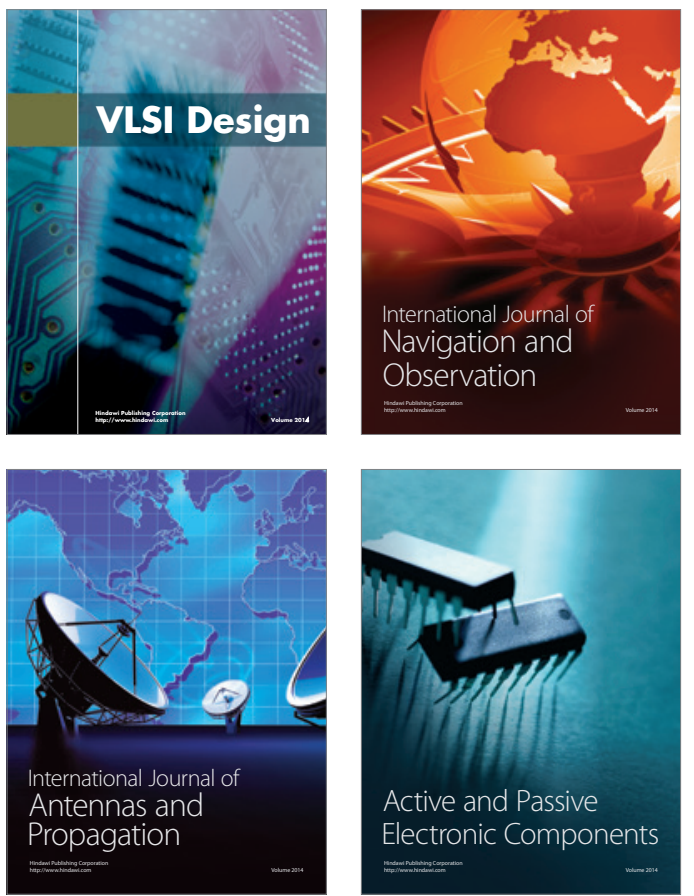
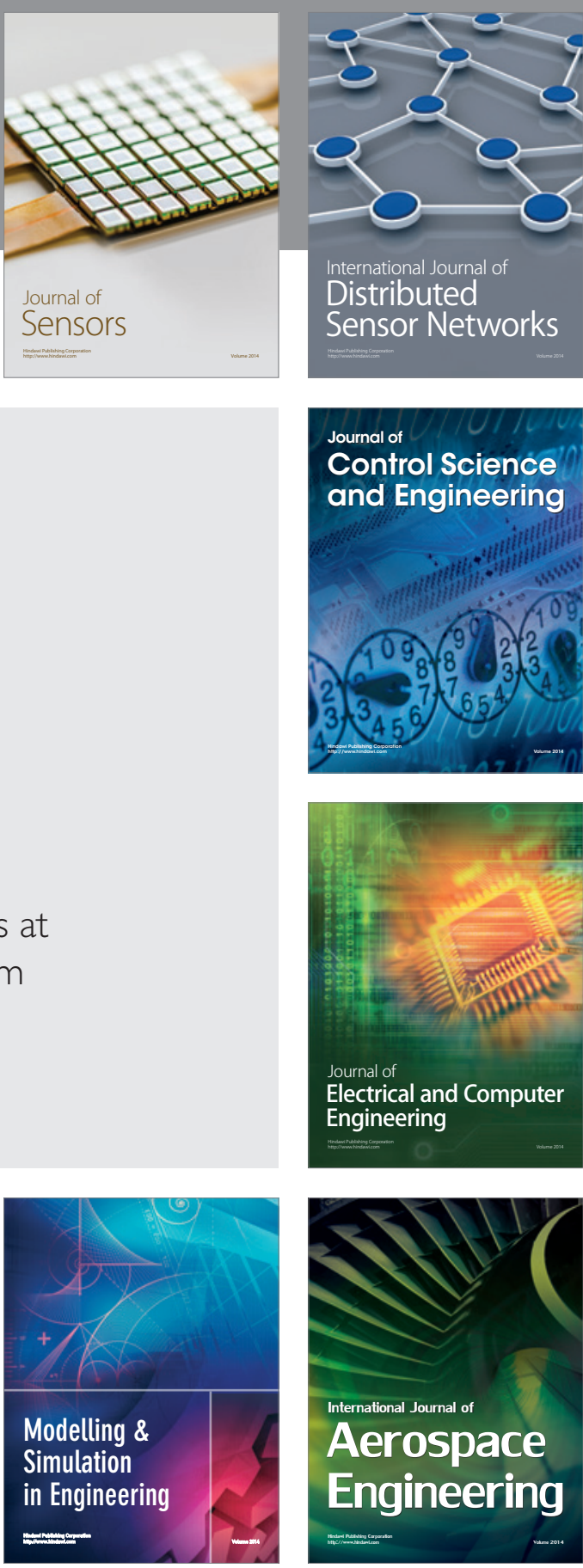

International Journal of

Distributed

Sensor Networks

Journal of

Control Science

and Engineering
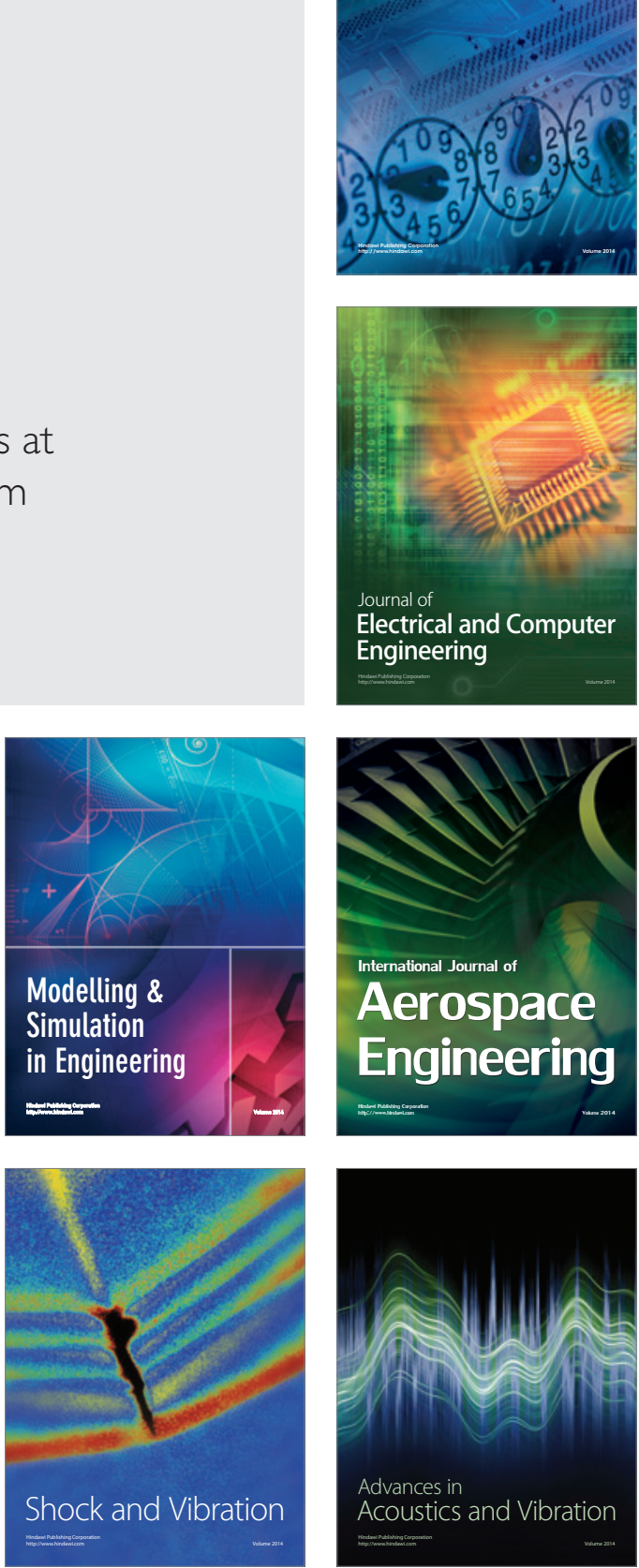